\title{
Online Time Delay Estimation in Networked Control Systems with Application to Bilateral Teleoperation
}

\author{
Kamil Cetin $^{a}$, Alper Bayrak ${ }^{b}$ and Enver Tatlicioglu ${ }^{\star c}$
}

\begin{abstract}
The problem of forward and backward time delays is significantly important for both control and feedback loop of networked control systems. These time delays give rise to latency in performance and thereby may destabilize the system. Therefore numerous methods have been proposed about time delay identification/estimation and compensation for networked control systems, especially for bilateral teleoperation systems. However, most compensation methods have been accomplished by considering offline time delay estimation for linear/nonlinear time delay control systems.

In this work, we propose an observer based estimation algorithm for round trip delay which is the sum of forward and backward time delays for a 1 degree-of-freedom nonlinear bilateral teleoperation system. Via Lyapunov based stability analysis, global boundedness of the observer errors along with their ultimate convergence and the convergence of the round trip delay estimator to the vicinity of its real value can be guaranteed in the closed-loop system. Finally, simulation and experimental studies are carried out utilizing the last link of a PHANToM Omni Haptic device moving like a one-link robot in the vertical plane.
\end{abstract}

\section{INTRODUCTION}

With the development of internet and global network, networked control systems have been attracting a lot of interest. Network based bilateral teleoperation can be considered as a subclass of networked control systems if it requires networked communication to close the loop between the local master and remote slave [1]. As a classical example of a bilateral teleoperation system, Figure 1 shows that a master robot handled by a human operator in a local station sends control inputs to a slave robot in a remote station and receives feedback from the remote site. These information are transmitted through a communication channel from the master robot to the slave robot and vice versa. Bilateral teleoperation systems have been applied in various areas such as mobile robots handling hazardous materials, telesurgery, underwater or space exploration [2]. Depending on the telerobotic application and the distance between master and slave robots, the use of communication media may cause loss of transparency, latency and instability [3]. In networked control systems, TCP/IP based communication is the most widespread architecture for a broad range of telerobotic applications. While TCP/IP architecture guarantees packet delivery with reliable transmission [4], [5], one of the most

${ }^{a} \mathrm{~K}$. Cetin is with the Department of Electrical \& Electronics Engineering, Gediz University, Izmir, Turkey.

${ }^{b}$ A. Bayrak is with the Department of Electrical \& Electronics Engineering, Abant Izzet Baysal University, Golkoy, Bolu, 14280 Turkey.

${ }^{c} \mathrm{E}$. Tatlicioglu is with the Department of Electrical \& Electronics Engineering, Izmir Institute of Technology, Urla, Izmir, 35430 Turkey.

${ }^{\star}$ To whom all the correspondence should be addressed. important problems in bilateral teleoperation systems with TCP/IP based communication channel is the existence of time delay. Depending on how signals are transmitted between the sources in bilateral teleoperation, time delay can be extensively classified into forward delay and backward delay. While the forward delay occurs in the slave robot when the control input delivered by the master robot, the backward delay occurs in the master robot when the measurement signal is fedback from the slave robot.

Numerous researchers have designed compensation methods for time delay in bilateral teleoperation over the last decades. In an early research [6], Anderson et al. developed passivity based scattering variables theory under constant time delay. Niemeyer et al. later extended the study by proposing a wave variables theory in [7]. In [8], Garcia et al. developed a Smith predictor based compensation method, then, in [9], extended the method by estimating the time delay online. However, these studies did not consider timevarying delays.

Time-varying delay compensation method was introduced by adopting the wave integrals structure in [10] and [11]. Considering the communication delay as a disturbance, in [12], [13] and [14], the authors proposed a time-varying delay compensation approach based on a network disturbance and a communication disturbance observer. Using a linear matrix inequality approach in [15], Chai et al. presented a memory observer based controller with adaptation to time delays. In [16] and [17], the authors developed a sliding mode observer to estimate the slave position on the master side and an extended Kalman Filter observer to estimate load torque and parameter variations on the slave side. In [18] and [19], the authors developed a predictor type sliding mode observer to use in conjunction with a disturbance observer. Delgado and Barreiro presented the stability of linear teleoperation systems with time-varying delay in [20]. Although the above mentioned methods compensate for time delay in bilateral teleoperation considering the communication delay as a disturbance, only a few of them focus on the identification of time delay. However, they focused on linear bilateral teleoperation systems with time delay.

Some other part of past research has proposed methods for nonlinear bilateral teleoperation systems with time delay. Liu and Chopra presented a set-point controller to compensate for time-varying delay for robotic manipulators [21] and [22], and developed further improvements considering the compensation of the gravitational torque in [23]. A sliding mode impedance controller was proposed for nonlinear bilateral teleoperation but under constant time delay in [24] and [25]. 


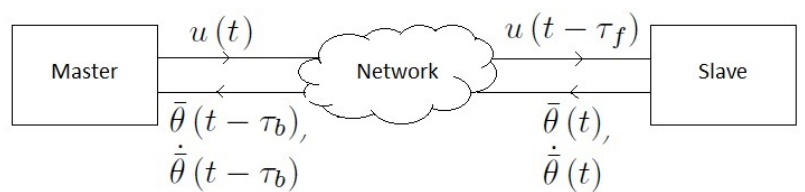

Fig. 1. The architecture of the networked bilateral teleoperation system

Review of the relevant past research highlights the fact that most of the works considered known communication induced delays when designing controllers. While there are some past works that aimed identification/estimation of communication induced delays, almost all of them were offline (i.e., the identification scheme was run after the experimental data was collected). In a novel departure from the existing past works on teleoperation systems, in this work, we propose an online round trip delay estimation method for networked control systems which includes a bilateral teleoperation application. First, the model of the overall system is given where the model of a one degree of freedom (dof) robot was utilized. Next, by treating the round trip delay as a nonlinear-like parameter, the estimation scheme is presented. The boundedness of the closed-loop system signals are demonstrated via Lyapunov arguments, while the convergence of the time delay estimator is also achieved. Simulation and experiments were conducted by considering the last link of a PHANToM Omni robot arm in the vertical plane.

The rest of the paper is organized as follows. In Section II, the dynamic model and state space form are described for one degree of freedom robot manipulator and the round trip delay is represented. In Section III, design of the round trip delay estimator is presented. Section IV introduces the Lyapunov based stability analysis. Simulation and experimental verifications are performed in Section V, and concluding remarks are given in Section VI.

\section{Networked Control System Model}

The dynamic model of a remotely located one degree of freedom robot has the following form [26]

$$
m l^{2} \ddot{\ddot{\theta}}(t)+m g l \sin (\bar{\theta}(t))+f \operatorname{sgn}(\dot{\bar{\theta}}(t))=u\left(t-\tau_{f}\right)
$$

where $\bar{\theta}(t), \dot{\bar{\theta}}(t), \ddot{\bar{\theta}}(t) \in \mathbb{R}$ are angular position, velocity and acceleration, respectively, $m \in \mathbb{R}$ is the mass of the link, $l \in \mathbb{R}$ is the length of the link, $g \in \mathbb{R}$ is the gravitational acceleration, $f \in \mathbb{R}$ is the friction constant, $\operatorname{sgn}(\cdot) \in \mathbb{R}$ is the standard signum function, and $u\left(t-\tau_{f}\right) \in \mathbb{R}$ is the control input torque that is delayed due to the communication line by an amount of $\tau_{f}$ seconds (i.e., forward delay).

As illustrated in Figure 1, it is considered that angular position and velocity of the one link robot are transmitted back and are subject to delays in the communication line in the sense that $\bar{\theta}\left(t-\tau_{b}\right)$ and $\dot{\bar{\theta}}\left(t-\tau_{b}\right)$ are received where $\tau_{b}$ is the backward time delay.

We now re-define the received angular position and velocity as

$$
\theta(t) \triangleq \bar{\theta}\left(t-\tau_{b}\right), \dot{\theta}(t) \triangleq \dot{\bar{\theta}}\left(t-\tau_{b}\right) .
$$

In view of (1), $\theta(t)$ and $\dot{\theta}(t)$ are generated from

$$
m l^{2} \ddot{\theta}(t)+m g l \sin (\theta(t))+f \operatorname{sgn}(\dot{\theta}(t))=u\left(t-\tau_{f}-\tau_{b}\right) .
$$

The round trip delay, denoted by $\tau_{r}$, is defined to be equal to the sum of $\tau_{f}$ and $\tau_{b}$. It should be highlighted that forward and backward delays are uncertain. We assumed that they are approximately equal since TCP/IP based round trip communication was used [27], [28]. The uncertain round trip time delay $\tau_{r}$ is bounded in the sense that $\tau_{r} \in R_{\tau}$ with the region is defined as $R_{\tau}=\left[\tau_{\min }, \tau_{\max }\right]$ where $\tau_{\min }$ and $\tau_{\max }$ are known lower and upper bounds, respectively.

Given the dynamic model in (3), the research problem attacked in this work is to estimate the uncertain round trip delay $\tau_{r}$ provided that model parameters are known, $\theta(t)$ and $\dot{\theta}(t)$ are available along with $u(t)$ and its past values being also available.

The model in (3) can alternatively be rewritten in state space form after defining $x_{1}(t) \triangleq \theta(t)$ and $x_{2}(t) \triangleq \dot{\theta}(t)$ in the following form

$$
\begin{aligned}
& \dot{x}_{1}=x_{2} \\
& \dot{x}_{2}=h\left(x_{1}, x_{2}, t, \tau_{r}\right)
\end{aligned}
$$

where $h\left(x_{1}, x_{2}, t, \tau_{r}\right) \in \mathbb{R}$ is defined as

$$
h \triangleq-\frac{g}{l} \sin \left(x_{1}(t)\right)-\frac{f}{m l^{2}} \operatorname{sgn}\left(x_{2}(t)\right)+\frac{1}{m l^{2}} u\left(t-\tau_{r}\right) .
$$

The nonlinear function $h$ is assumed to be either concave or convex in a region $R_{h}=[\underline{\tau}, \bar{\tau}]$ of $\tau_{r}$ that includes $R_{\tau}$ (i.e., $\left.0<\underline{\tau} \leq \tau_{\min } \leq \tau_{r} \leq \tau_{\max } \leq \bar{\tau}\right)$.

Assumption 1: The state vector $x \triangleq\left[x_{1}, x_{2}\right]^{T} \in \mathbb{R}^{2}$ is continuous, bounded, and Lipschitz in time in the following sense

$$
\left\|x\left(t_{1}\right)-x\left(t_{2}\right)\right\| \leq L_{1}\left|t_{1}-t_{2}\right| \quad \forall t_{1}, t_{2} \in \mathbb{R}^{+}
$$

where $L_{1} \in \mathbb{R}$ is a positive Lipschitz constant, and $t_{1}$ and $t_{2}$ are time instants. The nonlinear function $h$ is assumed to be Lipschitz with respect to its arguments in the following manner

$$
\left|h\left(t_{1}\right)-h\left(t_{2}\right)\right| \leq L_{2}\left(\| x\left(t_{1}\right)-x\left(t_{2}\right)||+\left|\tau_{0}\left(t_{1}\right)-\tau_{0}\left(t_{2}\right)\right|\right)
$$

for some time-varying function $\tau_{0}$ where $L_{2} \in \mathbb{R}$ is a positive Lipschitz constant.

\section{Design of Round Trip Delay Estimator}

The subsequent delay estimation algorithm will be designed by using position and velocity observer signals, denoted by $\hat{x}_{1}(t), \hat{x}_{2}(t) \in \mathbb{R}$, respectively, and are updated according to

$$
\begin{aligned}
& \dot{\hat{x}}_{1}=-k \tilde{x}_{1}+\hat{x}_{2} \\
& \dot{\hat{x}}_{2}=-\alpha \tilde{x}_{2}+\left(\alpha \varepsilon-a^{*}\right) \operatorname{sat}\left(\frac{1}{\varepsilon} \tilde{x}_{2}\right)+\hat{h}
\end{aligned}
$$

where $k, \alpha \in \mathbb{R}$ are constant positive observer gains, $\varepsilon \in \mathbb{R}$ is the desired precision of the subsequently designed round trip delay estimation algorithm, $a^{*}(t) \in \mathbb{R}$ is the tuning function 
that is yet to be designed, $\hat{h} \triangleq h\left(x_{1}, x_{2}, t, \hat{\tau}_{r}\right)$ with $\hat{\tau}_{r}(t)$ being the subsequently designed estimate of the uncertain round trip delay $\tau_{r}$, and $\tilde{x}_{1}(t), \tilde{x}_{2}(t) \in \mathbb{R}$ are observer errors defined as

$$
\begin{aligned}
& \tilde{x}_{1} \triangleq \hat{x}_{1}-x_{1} \\
& \tilde{x}_{2} \triangleq \hat{x}_{2}-x_{2}
\end{aligned}
$$

and $\operatorname{sat}(\cdot)$ is the standard saturation function defined as

$$
\operatorname{sat}(z)= \begin{cases}\operatorname{sgn}(z) & , \quad|z| \geq 1 \\ z & , \quad 1>|z| .\end{cases}
$$

The dynamics of the observer errors in (11) and (12) are obtained as

$$
\begin{aligned}
& \dot{\tilde{x}}_{1}=-k \tilde{x}_{1}+\tilde{x}_{2} \\
& \dot{\tilde{x}}_{2}=-\alpha \tilde{x}_{2}+\left(\alpha \varepsilon-a^{*}\right) \operatorname{sat}\left(\frac{1}{\varepsilon} \tilde{x}_{2}\right)+\hat{h}-h
\end{aligned}
$$

where (4), (9) and (12) were utilized to obtain the first expression and (5) and (10) were utilized to obtain the second expression.

The adaptive estimation rule for the round trip delay is designed as

$$
\dot{\hat{\tau}}_{r}=-\gamma \tilde{x}_{\varepsilon} \phi^{*}
$$

where $\gamma$ is a positive constant gain, $\phi^{*}(t)$ is a sensitivity function that is yet to be designed, and $\tilde{x}_{\varepsilon}(t) \in \mathbb{R}$ is an auxiliary error defined as

$$
\tilde{x}_{\varepsilon} \triangleq \tilde{x}_{2}-\varepsilon \operatorname{sat}\left(\frac{1}{\varepsilon} \tilde{x}_{2}\right) .
$$

While the adaptive time delay estimation rule is proposed as in $(16), \hat{\tau}_{r}(t)$ is required to remain on a known bounded set, to ensure this, the estimation rule is to be utilized with a projection algorithm that may be of the following form

$$
\hat{\tau}_{r}=\left\{\begin{array}{lll}
\tau_{\max } & \text { if } & \hat{\tau}_{r}>\tau_{\max } \\
\hat{\tau}_{r} & \text { if } & \tau_{\max } \geq \hat{\tau}_{r} \geq \tau_{\min } \\
\tau_{\min } & \text { if } & \tau_{\min }>\hat{\tau}_{r} .
\end{array}\right.
$$

As noted in [29], an adaptive law with a projection algorithm defined on a convex set retains the properties of the adaptive law without the projection algorithm.

The tuning function $a^{*}(t)$ introduced in (10) and the sensitivity function $\phi^{*}(t)$ introduced in (16) are found from the solutions of the following min-max optimization problem [30]

$$
\begin{aligned}
a^{*} & =\min _{\phi} \max _{\tau \in R_{h}}\left\{\operatorname{sat}\left(\frac{1}{\varepsilon} \tilde{x}\right)\left[\hat{h}-h-\gamma \tilde{\tau}_{r} \phi\right]\right\} \\
\phi^{*} & =\arg \min _{\phi} \max _{\tau \in R_{h}}\left\{\operatorname{sat}\left(\frac{1}{\varepsilon} \tilde{x}\right)\left[\hat{h}-h-\gamma \tilde{\tau}_{r} \phi\right]\right\}
\end{aligned}
$$

where $\tilde{\tau}_{r}(t) \in \mathbb{R}$ is the round trip delay estimation error

$$
\tilde{\tau}_{r} \triangleq \hat{\tau}_{r}-\tau_{r}
$$

The solutions of the optimization problem in (19) and (20) for $a^{*}(t)$ and $\phi^{*}(t)$ are obtained for two sub-cases as [30],
[31]. In case 1 , for $\tilde{x}_{2}(t) \geq 0$,

$$
\begin{aligned}
a^{*} & = \begin{cases}a_{1} & \text { if } h \text { is concave on } R_{h} \\
0 & \text { if } h \text { is convex on } R_{h}\end{cases} \\
\phi^{*} & = \begin{cases}\phi_{1} & \text { if } h \text { is concave on } R_{h} \\
\left.\frac{\partial h}{\partial \tau_{r}}\right|_{\tau_{r}=\hat{\tau}_{r}} & \text { if } h \text { is convex on } R_{h}\end{cases}
\end{aligned}
$$

and in case 2 , for $0>\tilde{x}_{2}(t)$,

$$
\begin{aligned}
a^{*} & = \begin{cases}0 & \text { if } h \text { is concave on } R_{h} \\
a_{1} & \text { if } h \text { is convex on } R_{h}\end{cases} \\
\phi^{*} & = \begin{cases}\left.\frac{\partial h}{\partial \tau_{r}}\right|_{\tau_{r}=\hat{\tau}_{r}} & \text { if } h \text { is concave on } R_{h} \\
\phi_{1} & \text { if } h \text { is convex on } R_{h}\end{cases}
\end{aligned}
$$

where $a_{1}$ and $\phi_{1}$ are scalar time-varying functions obtained from

$$
\begin{gathered}
a_{1}=\frac{(\hat{h}-\underline{h})\left(\hat{\tau}_{r}-\bar{\tau}\right)-(\hat{h}-\bar{h})\left(\hat{\tau}_{r}-\underline{\tau}\right)}{\beta(\bar{\tau}-\underline{\tau})} \\
\phi_{1}=\frac{\bar{h}-\underline{h}}{\gamma(\bar{\tau}-\underline{\tau})}
\end{gathered}
$$

where $\underline{h} \triangleq h\left(x_{1}, x_{2}, t, \underline{\tau}\right), \bar{h} \triangleq h\left(x_{1}, x_{2}, t, \bar{\tau}\right)$, and $\beta \in \mathbb{R}$ is defined as follows

$$
\beta= \begin{cases}1 & \text { if } h \text { is convex on } R_{h} \\ -1 & \text { if } h \text { is concave on } R_{h} .\end{cases}
$$

\section{Stability ANALYSis}

In this section, stability of observers and round trip delay estimation algorithm are analyzed in two sub-parts. In the first part, we ensure global boundedness of the observer errors along with their convergence. While, in the second part, convergence of the round trip delay estimator is to be analyzed.

Theorem 1: The observers in (9) and (10) and the round trip delay update law in (16) guarantee stability of the observer errors and the global boundedness of the overall system.

Proof: The subsequent Lyapunov type stability analysis will be done on the velocity observation error $\tilde{x}_{2}$ and the time delay estimation error $\tilde{\tau}_{r}$. From the structure of (15), if the difference between $h$ and its estimate $\hat{h}$ can be made small via the design of the round trip delay estimator in (16), then the velocity observer error (i.e., $\tilde{x}_{2}$ ) could also be made small. And from the structure of (14), when the velocity observer error is made small, then the position observer error (i.e., $\tilde{x}_{1}$ ) can be made small (that can be adjusted arbitrarily small by adjusting observer gain $k$ ).

Let $V\left(\tilde{x}_{\varepsilon}, \tilde{\tau}_{r}\right)$ be the following nonnegative function

$$
V \triangleq \frac{1}{2} \tilde{x}_{\varepsilon}^{2}+\frac{1}{2} \tilde{\tau}_{r}^{2}
$$

The time derivative of the Lyapunov function in (29) is found as

$$
\dot{V}=\tilde{x}_{\varepsilon}\left(\dot{\tilde{x}}_{\varepsilon}-\gamma \tilde{\tau}_{r} \phi^{*}\right)
$$

where (16) was utilized. Two cases on $\tilde{x}_{2}$ will be considered. When $\left|\tilde{x}_{2}\right|<\varepsilon$, since sat $\left(\frac{1}{\varepsilon} \tilde{x}_{2}\right)=\frac{1}{\varepsilon} \tilde{x}_{2}$, from its definition in (17), $\tilde{x}_{\varepsilon}=0$ and thus $\dot{V}=0$. When $\left|\tilde{x}_{2}\right| \geq \varepsilon$, since 
$\varepsilon \operatorname{sat}\left(\frac{1}{\varepsilon} \tilde{x}_{2}\right)=\varepsilon \operatorname{sgn}\left(\tilde{x}_{2}\right)$, then from the time derivative of (17), $\dot{\tilde{x}}_{\varepsilon}=\dot{\tilde{x}}_{2}$. Utilizing (15), along with (30) yields

$$
\dot{V}=-\alpha \tilde{x}_{\varepsilon}^{2}+\tilde{x}_{\varepsilon}\left[-a^{*} \operatorname{sat}\left(\frac{1}{\varepsilon} \tilde{x}_{2}\right)+\hat{h}-h-\gamma \tilde{\tau}_{r} \phi^{*}\right]
$$

where (17) was utilized. For this case, sat $\left(\frac{1}{\varepsilon} \tilde{x}_{2}\right)=$ $\operatorname{sgn}\left(\tilde{x}_{2}\right)=\operatorname{sgn}\left(\tilde{x}_{\varepsilon}\right)$ is valid. In view of this, (31) can be rewritten as

$$
\begin{aligned}
\dot{V} & =-\alpha \tilde{x}_{\varepsilon}^{2} \\
& +\tilde{x}_{\varepsilon} \operatorname{sat}\left(\frac{1}{\varepsilon} \tilde{x}_{2}\right)\left[\operatorname{sat}\left(\frac{1}{\varepsilon} \tilde{x}_{2}\right)\left(\hat{h}-h-\gamma \tilde{\tau}_{r} \phi^{*}\right)-a^{*}\right] .
\end{aligned}
$$

Since $\tilde{x}_{\varepsilon}$ sat $\left(\frac{1}{\varepsilon} \tilde{x}_{2}\right)=\left|\tilde{x}_{\varepsilon}\right|$, when

$$
a^{*}>\operatorname{sat}\left(\frac{1}{\varepsilon} \tilde{x}_{2}\right)\left(\hat{h}-h-\gamma \tilde{\tau}_{r} \phi^{*}\right)
$$

is satisfied then

$$
\dot{V} \leq-\alpha \tilde{x}_{\varepsilon}^{2}
$$

Therefore, $a^{*}$ is to be maximized in the sense that

$$
a^{*}=\max _{\tau \in R_{h}}\left\{\operatorname{sat}\left(\frac{1}{\varepsilon} \tilde{x}_{2}\right)\left(\hat{h}-h-\gamma \tilde{\tau}_{r} \phi^{*}\right)\right\}
$$

for any $\phi^{*}$. But, since usually lower gains are preferred, finding $\phi^{*}$ that minimizes (35) yields

$$
a^{*}=\min _{\phi} \max _{\tau \in R_{h}}\left\{\operatorname{sat}\left(\frac{1}{\varepsilon} \tilde{x}_{2}\right)\left(\hat{h}-h-\gamma \tilde{\tau}_{r} \phi^{*}\right)\right\} \text {. }
$$

From (29) and (34), it is clear that $V\left(\tilde{x}_{\varepsilon}, \tilde{\tau}_{r}\right) \in \mathcal{L}_{\infty}$ and thus $\tilde{x}_{\varepsilon}, \tilde{\tau}_{r} \in \mathcal{L}_{\infty}$. From its definition in (17), it is easy to obtain that $\tilde{x}_{2} \in \mathcal{L}_{\infty}$, and from (14), it is easy to see that $\tilde{x}_{1}, \dot{\tilde{x}}_{1} \in \mathcal{L}_{\infty}$. From (16), boundedness of $\dot{\hat{\tau}}_{r}$ is also ensured. It is then easy to show that all the signals on the right hand side of (15) remain bounded (i.e., $\dot{\tilde{x}}_{2} \in \mathcal{L}_{\infty}$ ), and from the time derivative of (17), one can prove that $\dot{\tilde{x}}_{\varepsilon} \in \mathcal{L}_{\infty}$. Standard signal chasing arguments can then be utilized to prove global boundedness of all the signals under the closed-loop operation. Furthermore, after integrating (34) in time from the initial time to infinity, one can demonstrate $\tilde{x}_{\varepsilon} \in \mathcal{L}_{2}$. Since $\tilde{x}_{\varepsilon}, \dot{\tilde{x}}_{\varepsilon} \in \mathcal{L}_{\infty}$, Barbalat's Lemma in [32] can be utilized to demonstrate that $\left|\tilde{x}_{\varepsilon}\right| \rightarrow 0$. From its definition in (17), it is easy to see that when $\left|\tilde{x}_{\varepsilon}\right| \rightarrow 0$ then $\left|\tilde{x}_{2}\right|$ remains less than $\varepsilon$, and after solving the linear differential inequality in (14), one can find that $\left|\tilde{x}_{1}\right|$ remains less than $\frac{\varepsilon}{k}$.

Theorem 2: The round trip delay estimation error $\tilde{\tau}_{r}(t)$ is ensured to remain in a known bound which can be made arbitrarily small provided that the following nonlinear persistency of excitation like term

$$
\beta\left(t_{2}\right)\left[h\left(x_{1}\left(t_{2}\right), x_{2}\left(t_{2}\right), t_{2}, \hat{\tau}_{r}\left(t_{1}\right)\right)-h\left(x_{1}\left(t_{2}\right), x_{2}\left(t_{2}\right), t_{2}, \tau_{r}\right)\right.
$$

is greater than $\varepsilon_{u}\left|\hat{\tau}_{r}\left(t_{1}\right)-\tau_{r}\right|$ where $\varepsilon_{u}$ is a positive constant with $t_{2}>t_{1}>t_{0}$.

Proof: After ensuring the boundedness of all the signals under the closed-loop operation, the convergence of the round trip delay estimator is investigated via detailed analysis. The reader is referred to [30] for a similar convergence proof.

\section{Numerical Studies}

The performance of the proposed round trip delay estimator was evaluated by numerical studies where PHANToM Omni haptic device was used. The PHANToM Omni haptic device, as shown in Figure 2, can give position in 6 degrees-of-freedom consisting of three translational and three rotational directions. The device has two links, three active joints and three passive wrist joints connected to the end of the second link. Since only the first three joints of the device are actuated, it generates haptic force feedback on the translational directions. In the simulation and experimental studies, considering a remotely controlled one degree of freedom robot model in the vertical plane, we apply the torque control input only to the last link of the the device in the vertical plane by releasing the first two links at their own positions.

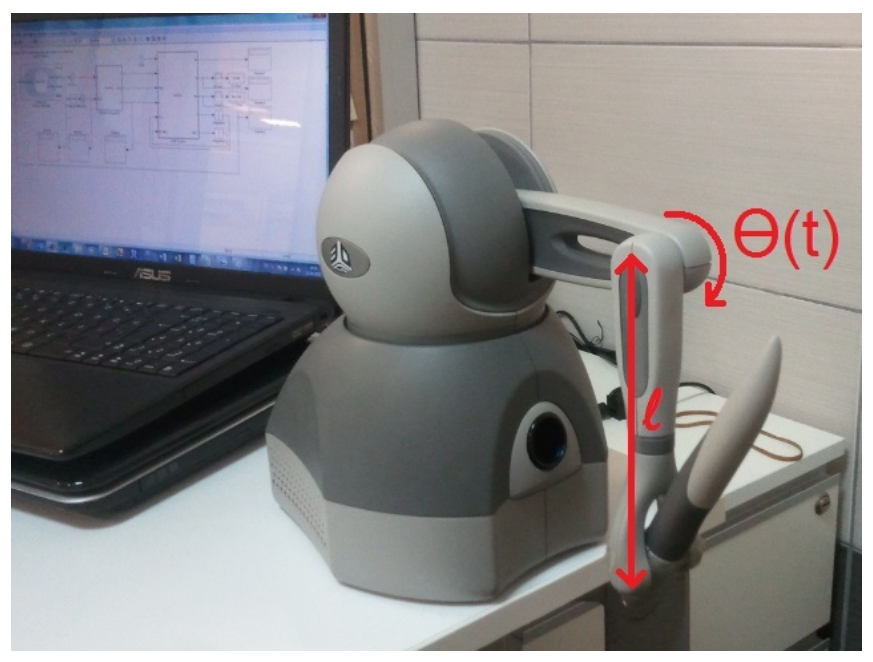

Fig. 2. PHANToM Omni haptic device

\section{A. Simulation Results}

The proposed round trip delay estimator was simulated on PHANToM Omni haptic device by considering the last link in the vertical plane as in (3) where the link length is $l=0.135 \mathrm{~m}$, the link mass is $m=0.265 \mathrm{~kg}$, the friction constant is $f=0.065 \mathrm{Nm}$, and the acceleration of gravity is $g=9.81 \mathrm{~m} / \mathrm{s}^{2}$ were experimentally obtained.

During the simulation studies, the applied torque input from the master site to the one dof robot link in the slave site is $0.3 \sin (2 \pi t)$. Simulation studies run on MATLAB Simulink with variable step size.

The simulation was run for $100 \mathrm{sec}$. The update rule for time delay estimation in (16) was utilized with the gains $\varepsilon=1 e-6, \alpha=50$ and $\gamma=0.008$. The upper and lower bounds of time delay was chosen as 0 and 2 seconds, respectively. The actual forward and backward time delays in communication line were determined as equal and $0.953 \mathrm{sec}$. for the simulation.

The simulation result is given in Figure 3. It should be highlighted that the communication delays are bilateral, so the delays get double. Figure 3 shows that the proposed 


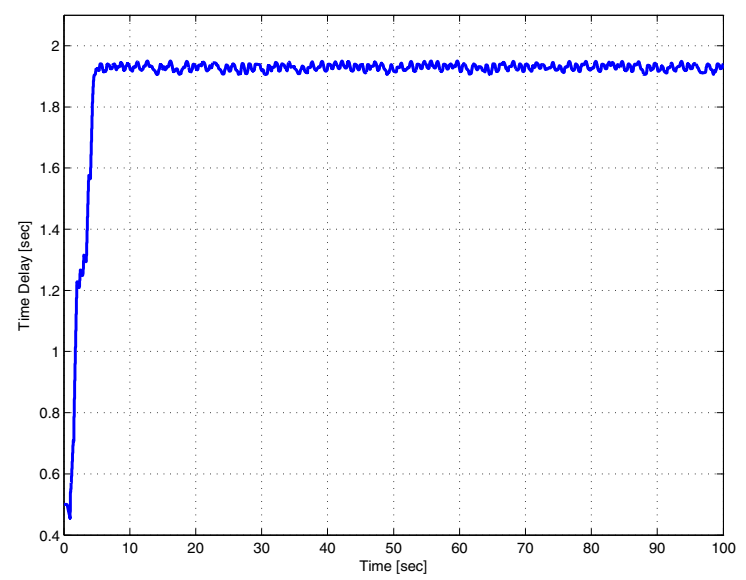

Fig. 3. Time delay estimation for the simulation.

estimator computes the round trip delay around $1.9 \mathrm{sec}$. which is very close to the actual round trip delay $1.906 \mathrm{sec}$. From Figures 3 , it is clear that successive estimation result was achieved for the proposed online round trip delay estimator.

\section{B. Experimental Results}

During the experimental studies, for the communication interface, LAN (local area network) port is used between the device and a computer, and OpenHaptics ToolKit allows realtime applications on the computer. Experimental studies run on MATLAB Simulink with a data rate of $1000 \mathrm{~Hz}$. In realtime experimental application, we utilized Phantom Toolbox in MATLAB Simulink Quarc Library to transmit torques in the joint-space to the Phantom device, and to receive the joint angles of the device. Communication between the master and slave sites was done using TCP/IP sockets. Therefore, TCP/IP might result in packet delay but not packet loss [4].

The system was run for $100 \mathrm{sec}$. for the experiment. The applied torque input from the master site to the last link of the device in the slave site is $0.3 \sin (2 \pi 0.1 t)$. The update rule for time delay estimation in (16) was utilized with the gains $\varepsilon=1 e-9, \alpha=400$ and $\gamma=0.1$. The upper and lower bounds of time delay was chosen as 0 and 3.5 seconds. The actual forward and backward time delays in communication line were measured as, approximately, equal and $0.953 \mathrm{sec}$. for this experiment. Since the communication delays are bilateral, so the actual round trip delay becomes 1.906 sec.

The experimental result is given in Figure 4. Figure 4 shows that the proposed estimator computes the round trip delay around $1.9 \mathrm{sec}$. which is very close to the actual round trip delay after the $40^{t h}$ sec. As can be seen from the Figure 4, successive estimation result was achieved in the experimental study.

\section{CONCLUSIONS}

In this work, our main contribution is the development and numerical evaluation of online round trip delay esti-

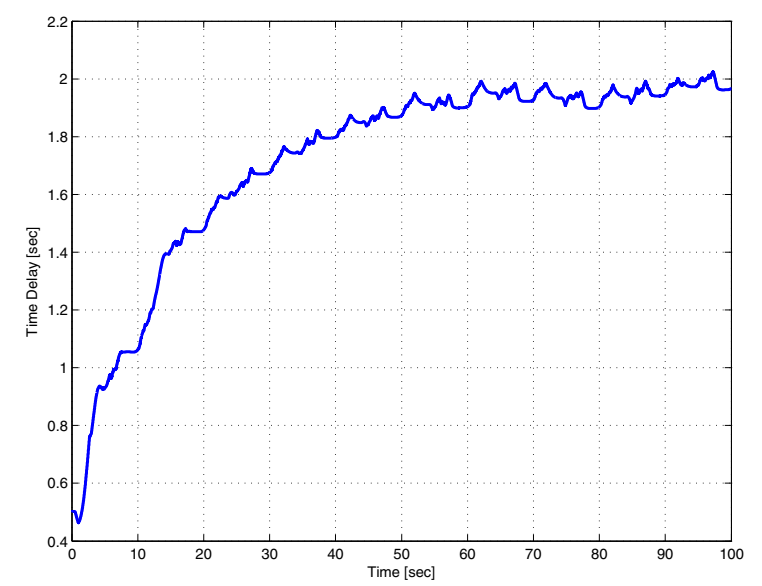

Fig. 4. Time delay estimation for the experiment.

mation method for networked control systems. First, we designed the position and velocity observer signals from the dynamic models of the nonlinear bilateral teleoperation system. The estimation algorithm for the round trip delay was then proposed utilizing the dynamics of the observer errors. According to the proposed methodology, when the slave site transmitted back the position and velocity signals with the backward time delay, the master site generates the control input estimating online round trip delay. The stability analysis was performed to ensure global boundedness of the observer errors along with their convergence and the convergence of the round trip delay estimator. The validity of the proposed method was verified by simulation and experimental studies utilizing the last link of the PHANToM Omni haptic device moving in the vertical plane.

In the future work, the proposed method will be applied into nonlinear control techniques to compensate the time delay in bilateral teleoperation applications.

\section{ACKNOWLEDGMENTS}

Works of K. Cetin and E. Tatlicioglu are supported in part by The Scientific and Technological Research Council of Turkey via grant number 113E147.

\section{REFERENCES}

[1] F. Lian, J. Moyne, and D. Tilbury, "Network design consideration for distributed control systems," IEEE Transactions on Control Systems Technology, vol. 10, no. 2, pp. 297-307, 2002.

[2] P. F. Hokayem and M. W. Spong, "Bilateral teleoperation: An historical survey," Automatica, vol. 42, no. 12, pp. 2035-2057, 2006.

[3] J.-P. Richard, "Time-delay systems: an overview of some recent advances and open problems," Automatica, vol. 39, no. 10, pp. 16671694, 2003.

[4] W. Zhang, M. Branicky, and S. Phillips, "Stability of networked control systems," Control Systems, IEEE, vol. 21, no. 1, pp. 84-99, Feb 2001 .

[5] D. Botturi, P. Fiorini, R. Muradore, and D. Quaglia, "Simulation of networked control systems with applications to telerobotics," in Control Conference (ECC), 2009 European, 2009, pp. 1481-1486.

[6] R. Anderson and M. Spong, "Bilateral control of teleoperators with time delay," Automatic Control, IEEE Transactions on, vol. 34, no. 5, pp. 494-501, 1989. 
[7] G. Niemeyer and J. Slotine, "Using wave variables for system analysis and robot control," in IEEE International Conference on Robotics and Automation, vol. 2, 1997, pp. 1619-1625.

[8] C. E. Garcia, R. Carelli, J. F. Postigo, and B. Morales, "Time delay compensation control structure for a robotic tele-operation system," in IFAC Conference SICICA 2000, Buenos Aires, 2000.

[9] E. Garcia, F. Postigo, A. Castro, R. Carelli, Kuchen, and B., "On-line estimation of communication time delay in a robotic teleoperation system," Latin American applied research, 2003.

[10] T. Zhang and Y. Li, "A control scheme for bilateral teleoperation systems based on time-varying communication delay identification," in 1st International Symposium on Systems and Control in Aerospace and Astronautics, 2006, pp. 6 pp.-278

[11] T. Zhang and Y.-Q. Cui, "A bilateral control of teleoperators based on time delay identification," in IEEE Conference on Robotics, Automation and Mechatronics, 2008, pp. 797-802.

[12] K. Natori, T. Tsuji, and K. Ohnishi, "Time delay compensation by communication disturbance observer in bilateral teleoperation systems," in 9th IEEE International Workshop on Advanced Motion Control, 2006, pp. 218-223.

[13] K. Natori, R. Oboe, and K. Ohnishi, "Stability analysis and practical design procedure of time delayed control systems with communication disturbance observer," IEEE Transactions on Industrial Informatics, vol. 4, no. 3, pp. 185-197, 2008.

[14] K. Natori, T. Tsuji, K. Ohnishi, A. Hace, and K. Jezernik, "Timedelay compensation by communication disturbance observer for bilateral teleoperation under time-varying delay," IEEE Transactions on Industrial Electronics, vol. 57, no. 3, pp. 1050-1062, 2010.

[15] L. Chai and S. Fei, "An improved approach of adaptive control for time-delay systems based on observer," in Advances in Neural Networks, ser. Lecture Notes in Computer Science, D. Liu, S. Fei, Z.-G. Hou, H. Zhang, and C. Sun, Eds. Springer Berlin Heidelberg, 2007, vol. 4491, pp. 222-230.

[16] B. Gadamsetty, S. Bogosyan, M. Gokasan, and A. Sabanovic, "Novel observers for compensation of communication delay in bilateral control systems," in 35th Annual Conference of IEEE Industrial Electronics, IECON'09., 2009, pp. 3019-3026.

[17] S. Bogosyan, B. Gadamsetty, M. Gokasan, A. Sabanovic, and M. Unel, "Experimental evaluation of sliding mode and ekf observers for network delay compensation in bilateral control," International Review of Electrical Engineering, vol. 5, no. 5, pp. 2484-2493, 2010.

[18] S. Dikyar, T. Leblebici, D. Ozcelik, M. Unel, A. Sabanovic, and S. Bogosyan, "Delay compensation for nonlinear teleoperators using predictor observers," in 36th Annual Conference on IEEE Industrial Electronics Society, 2010, pp. 1489-1494.

[19] T. Leblebici, B. Calli, M. Unel, A. Sabanovic, S. Bogosyan, and M. Gokasan, "Delay compensation in bilateral control using a sliding mode observer," Turkish Journal of Electrical Engineering \& Computer Sciences, vol. 19, no. 6, pp. 851-859, 2011.

[20] E. Delgado and A. Barreiro, "Stability of teleoperation systems for time-varying delays by lmi techniques," in Control Conference (ECC), 2007 European, 2007, pp. 4214-4221.

[21] Y.-C. Liu and N. Chopra, "A new architecture for set-point control of robotic manipulators with time-varying input/output delays," in ASME 2011 Dynamic Systems and Control Conference, 2011, pp. 129-136.

[22] — - "Control of robotic manipulators under input/output communication delays: Theory and experiments," IEEE Transactions on Robotics, vol. 28 , no. 3, pp. 742-751, 2012.

[23] _ _ "Gravity-compensation-driven position regulation for robotic systems under input/output delays," IEEE Transactions on Control Systems Technology, vol. 22, no. 3, pp. 995-1005, 2014.

[24] A. Vafaei and M. Yazdanpanah, "Terminal sliding mode impedance control for bilateral teleoperation under unknown constant time delay and uncertainties," in Control Conference (ECC), 2013 European, 2013, pp. 3748-3753.

[25] L. Ma, Z. Xu, and K. Schilling, "Robust bilateral teleoperation of a car-like rover with communication delay," in Control Conference (ECC), 2009 European, 2009, pp. 2337-2342.

[26] M. Spong and M. Vidyasagar, Robot Dynamics and Control. New York, NY, USA: John Wiley \& Sons Inc., 1989.

[27] A. Pathak, H. Pucha, Y. Zhang, Y. Hu, and Z. Mao, "A measurement study of internet delay asymmetry," in Passive and Active Network Measurement, ser. Lecture Notes in Computer Science. Springer Berlin Heidelberg, 2008, vol. 4979, pp. 182-191.
[28] Z. Li, Y. Xia, and C.-Y. Su, Intelligent Networked Teleoperation Control. Springer, 2015.

[29] P. Ioannou and J. Sun, Robust Adaptive Control. Englewood Cliffs, NJ, USA: Prentice Hall, 1996.

[30] A. Bayrak, "Online time delay identification and adaptive control for general classes of nonlinear systems," PhD dissertation, Izmir Institute of Technology, 2013.

[31] A. M. Annaswamy, F. P. Skantze, and A.-P. Loh, "Adaptive control of continuous time systems with convex/concave parametrization," Automatica, vol. 34, no. 1, pp. 33 - 49, 1998.

[32] M. Krstic, I. Kanellakopoulos, and P. Kokotovic, Nonlinear and Adaptive Control Design. New York, NY, USA: John Wiley \& Sons, 1995. 